\title{
Slip-stick excitation and travelling waves excite silo honking
}

\author{
Katarzyna Warburton ${ }^{1}$, Elze Porte ${ }^{2,3}$, and Nathalie Vriend ${ }^{1, \star}$ \\ ${ }^{1}$ University of Cambridge, UK, Department of Applied Mathematics and Theoretical Physics \\ ${ }^{2}$ Imperial College London, UK, Department of Mechanical Engineering \\ ${ }^{3}$ University of Twente, Netherlands, Department of Mechanical Engineering
}

\begin{abstract}
Silo honking is the harmonic sound generated by the discharge of a silo filled with a granular material. In industrial storage silos, the acoustic emission during discharge of PET-particles forms a nuisance for the environment and may ultimately result in structural failure. This work investigates the phenomenon experimentally using a laboratory-scale silo, and successfully correlates the frequency of the emitted sound with the periodicity of the mechanical motion of the grains. The key driver is the slip-stick interaction between the wall and the particles, characterized as a wave moving upwards through the silo. A quantitative correlation is established for the first time between the frequency of the sound, measured with an electret microphone, and the slip-frequency, measured with a high-speed camera. In the lower regions of the tube, both the slip-stick motion and the honking sound disappear.
\end{abstract}

\section{Introduction}

The discharge of industrial silos is known to produce low frequency resonance known as silo honking or silo $\mathrm{mu}$ sic $[1,3-5]$. The sound forms a nuisance for the environment when the structure starts to act as a loudspeaker. The acoustic emission consists of a main frequency (typically between $50-200 \mathrm{~Hz}$ ) and a fixed set of harmonics.

We model silo honking in a laboratory set-up with a small diameter vertical tube filled with fine particles, reproducing the key features of silo music [1]. A typical spectrogram is shown in figure 1 and shows two distinct resonances contributing to the honk: a falling and a rising one. When the distinct resonances intersect in frequency at a given time, the amplitude temporary increases. At irregular times jumps towards a higher frequency occur, usually representing an alteration of approximately $5 \mathrm{~Hz}$. A peculiar observation is that the duration of the acoustic emission is significantly shorter than the total duration of the discharge: the audible sound stops abruptly, well before the silo is emptied of particles.

The falling signal is the air resonance, not too dissimilar from an organ pipe, forming an open-closed tube system of the resonating air layer between the bulk surface and the top of the tube [6]:

$$
f_{n}=\frac{c}{4 H_{a}}(2 n-1),
$$

with air layer height $H_{a}$, air velocity $c$, and odd harmonics $(2 n-1=1,3,5 \ldots)$. The frequency scales inversely with the length, resulting in a decreasing frequency as the silo discharges and $H_{a}$ increases. The structural reso-

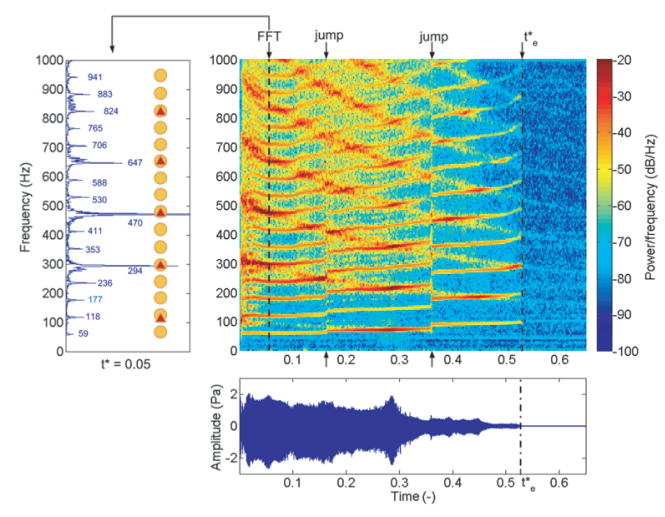

Figure 1. A typical spectrogram, signal and FFT plot [2], featuring the falling air and rising structural resonance and sudden jumps. Note that time has been non-dimensionalised by the total discharge time and time $t_{e}^{*}$ denotes the abrupt cessation of the sound, just past halfway through the discharge.

nance typically starts at a lower frequency, displays all integer harmonics $(n=1,2,3 \ldots)$ and the frequency increases slowly by a factor of around 1.5 during silo discharge. This structural resonance is not a result of the natural modes of the tube: the bending, radial-axial and longitudinal modes are excluded as the dominant mechanism for setting the structural frequency due to their incompatible harmonic structure and/or frequency range. It is the source of this structural resonance that we investigate in this paper.

\footnotetext{
^e-mail: n.m.vriend@damtp.cam.ac.uk
} 

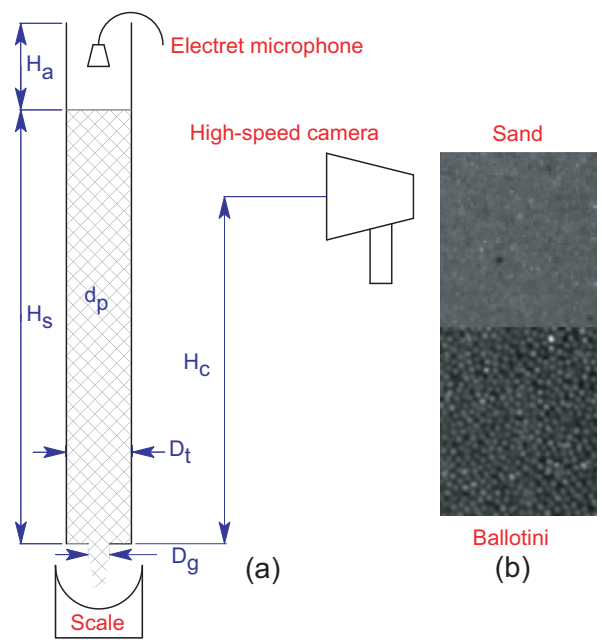

(b)

Figure 2. (a) A schematic of the experimental setup, and (b) images of grains taken by the camera (top, $0.15-0.25 \mathrm{~mm}$ sand, below, $0.3-0.4 \mathrm{~mm}$ ballotini).

\section{Methods}

\subsection{Experimental setup}

For our laboratory experiments, we model the silo as a $2 \mathrm{~m}$ long vertical tube (figure 2), filled with a fixed mass of $4 \mathrm{~kg}$ to a height of approximately $H_{s}=1.45 \mathrm{~m}$, which leaves an air layer $H_{a}$. We vary the wall material (perspex and glass) while keeping the inner tube diameter essentially constant ( $D_{t}=50 \mathrm{~mm}$ versus $D_{t}=51 \mathrm{~mm}$, respectively).

The tube is supported at the base by an MDF plate with outlet gaps of diameters $D_{g}$ between $8 \mathrm{~mm}$ and $14 \mathrm{~mm}$, which rests on an aluminium frame. The sides are attached to a rail via a padded strapping, allowing the tube to vibrate without being rigidly fixed to the supporting structure. The tube is filled to $H_{s}$ with granular material of different composition and size. In this work, we used sieved particles, of both silver sand $\left(d_{p}=150-250 \mu \mathrm{m}\right)$ and ballotini $\left(d_{p}=90-150 \mu m, 150-250 \mu m\right.$, or $\left.300-400 \mu m\right)$.

Above the grain layer, near the top of the tube, a small electret microphone with a sampling frequency of $44100 \mathrm{~Hz}$ is suspended, acquiring acoustical data for the duration of the discharge. The rate of discharge is derived by tracking the discharged mass with a set of Ohaus Scout Pro electronic scales. Video footage, collected at various heights $H_{c}$, is acquired by up to two Photon FASTCAM SA1.1 high-speed cameras through the transparent tube walls.

\subsection{Processing of camera footage}

The camera is mounted at various heights $H_{c}$ between $0.7 \mathrm{~m}$ and $1.1 \mathrm{~m}$ at $10 \mathrm{~cm}$ intervals, to image the particle motion at the walls at different stages of the discharge. Visual data is post-processed with three different modes: (1) tracking of the bulk surface, (2) particle tracking velocimetry routines to measure particle displacements, and (3) particle image velocimetry routines to measure flow structures. For mode (1), a wide-angle $24 \mathrm{~mm}$ lens was used to obtain a visible $50 \mathrm{~cm}$ region of the tube at $60 \mathrm{fps}$, showing pale grains against a black background. This image was used as input in a Sobel edge detection filter, and produced position of the free surface as a function of time. For mode (2) and (3), a $60 \mathrm{~mm}$ lens collected data at a higher frame rate and focussing on a much smaller area $(5$ $\mathrm{cm}$ by $1 \mathrm{~cm}$ ), to resolve particle motions and flow structure respectively. For mode (2), one camera at $2000 \mathrm{fps}$ was used, and for mode (3), two cameras, placed $50 \mathrm{~cm}$ apart at $H_{c}=0.65 \mathrm{~m}$ and $H_{c}=1.15 \mathrm{~m}$, were used at 5400 fps to measure the wave of motion by cross-correlation.

\section{Results and discussion}

\subsection{Structural resonance}

Typically, the first harmonic of the audio recording is initially in the range of $50-70 \mathrm{~Hz}$, varying slightly depending on atmospheric conditions and packing fraction. However, during discharge this first harmonic steadily increases, almost doubling its initial value by the time that sound abruptly ceases. Interestingly, changing the wall material did not alter the initial frequency significantly, even though the frictional characteristics between the particles and the wall material are quite different. The particle choice did make a difference: main harmonic for sand was initially approximately $20 \%$ higher than that for ballotini.

The integer harmonics $(\mathrm{n}=1,2,3, \ldots)$ and slowly rising frequency of the structural resonance (figure 1) indicate a closed-closed system of decreasing length or increasing velocity. Several authors $[4,7]$ suggested that the structural resonance is at a natural eigenmode frequency of the silo. Our initial work [2] demonstrated this explanation is inadequate due to the harmonic structure and the frequency range. To support this statement, we now performed proof-of-concept experiments with a PCB 352C22 accelerometer attached to different positions on the tube during discharge. No radial vibrations could be recorded for the perspex tube and only low amplitude vibrations were recorded with the thinner glass tube. The axial modes are probed by closing the base of the tube with a flexible latex sheet and simply tapping the filled tube through the sheet. Motion measured by the accelerometer in axial direction matched the characteristics of the structural resonance, but the lowest natural resonant frequency of our tube was $80 \mathrm{~Hz}$, which is too high to account for the silo honking.

\subsection{Slip-stick frequency}

As reported by Buick et al. [8] and Börzsönyi et al. [4], particles undergo periodic slip-stick motion down the tube, with extended sticking to the tube wall followed by acceleration at close to $g$. As seen from the displacement data (blue) in figure 3, each slip-stick cycle is extremely regular, and therefore it is possible to extract its recurrent frequency using temporal frequency analysis. Of note is the slight relaxation, resulting in a small upward displacement and small negative velocities, which occurs close to the bulk surface for early stages of the process for sand particles and the smaller ballotini sizes. In a novel approach, 


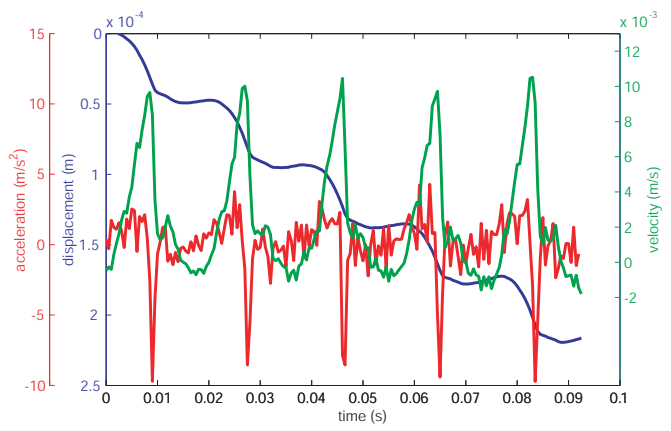

Figure 3. Average particle motion, velocity, and acceleration for ballotini particles in a glass tube at $H_{c}=1.1 \mathrm{~m}$. Each slip is around $0.05 \mathrm{~mm}$, with velocities reaching $10^{-3} \mathrm{~ms}^{-1}$, and deceleration around $g$.
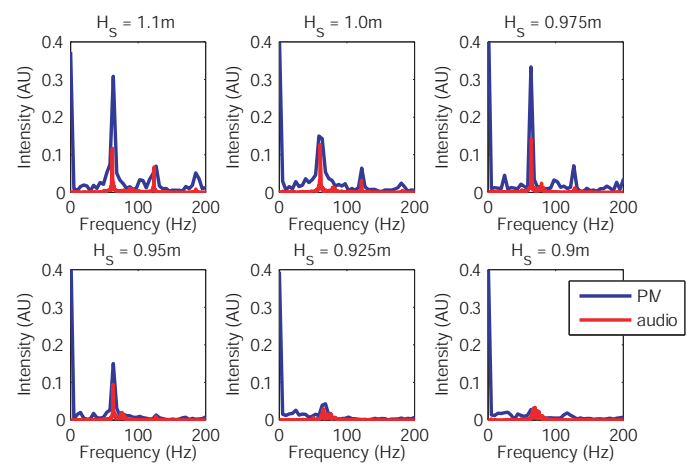

Figure 4. Comparison between the frequency of slip-stick particle motion and recorded audio, for ballotini particles in a perspex tube. The data was collected when the free surface of the sand moved from $H_{s}=1.1 \mathrm{~m}$ to $H_{s}=0.9 \mathrm{~m}$, while the camera was located at $H_{c}=H_{s}-0.05 \mathrm{~m}$ : the higher harmonics cut out first, and all sound and slip-stick motion ceases below $0.9 \mathrm{~m}$.

we capture simultaneously audio data and record particle motion to investigate the correlation between the two measurements. Figure 4 illustrates that the slip-stick frequency derived from particle motion and the audible structural resonance show an exact match within the available resolution of $0.5 \mathrm{~Hz}$ for a variety of stages in the release. This surprising result has not been reported before: previous work identified slip-stick as a characteristic of silo honking, but a quantitative correlation has never been established. The correspondence between slip-stick was unaffected by flow rate, particle type, or height of recording, and seen in every single run. As the free surface of the sand moved below $0.9 \mathrm{~m}$, both the slip-stick motion and the audible frequency were significantly reduced and disappeared.

\subsection{Slip-stick steps}

Important characteristics of slip-stick beyond the frequency are the step-size and the maximum and average velocity of motion. The rate of discharge through the base gap, measured with a scale, is not periodic but continuous. The slip-stick behavior gradually disappears as the free surface drops to heights below $H_{s}=0.9 \mathrm{~m}$, only occurs to a small extent below the critical height at which honking cuts out in early parts of the experiment and is

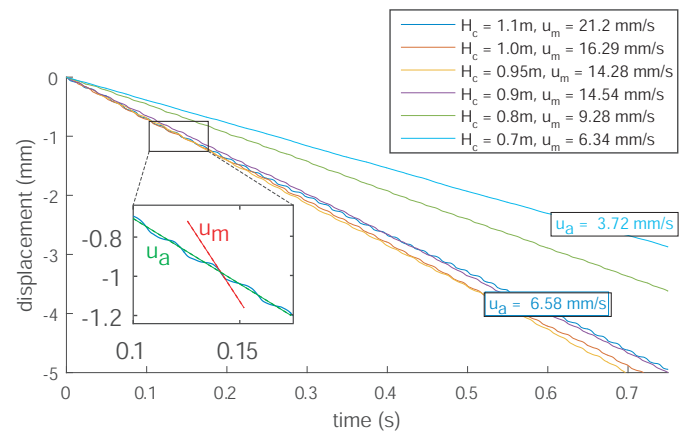

Figure 5. Initial particle motion for ballotini on perspex at different heights (plotted on the right hand axis). Little slip-stick motion is observed at $0.8 \mathrm{~m}$ and below. Note also that particles at the top of the tube initially move faster, as seen in the varying gradient: $u_{a}=6.58 \mathrm{mms}^{-1}$ for $H_{c}=1.1 \mathrm{~m}$ to $u_{a}=3.72 \mathrm{mms}^{-1}$ for $H_{c}=0.7 \mathrm{~m}$.

always absent in the lower regions of the tube. This indicates that the upper portion of column of particles has the largest role in exciting silo honking.

Figure 5 shows that average velocity of the grains at the side-wall, whether they undergo slip-stick or not, is initially approximately constant around $u_{a}=7 \mathrm{mms}^{-1}$, but drops dramatically by a factor 2 for much lower heights of the free surface. As the overall discharge rate does not change, we attribute this to a different internal velocity profile, where in the lower regions of the tube larger continuous shear rates occur close to the wall, where in the upper regions these large shear rates are only intermittent. This assessment is further strengthened by the observation that the instantaneous maximum velocity during the slipportion of the motion is very high (up to $u_{m}=21 \mathrm{mms}^{-1}$ ) initially at $H_{c}=1.1 \mathrm{~m}$, but consistently decreases with height, by a factor of 3.5 at heights of $H_{c}=0.7 \mathrm{~m}$. This can be visualized by the fact that for the upper regions, the velocity fluctuations and maximum velocity peak are high, but spread over a small time step size, while for the lower regions, the maximum velocity is much lower and smeared out over time. This process is a continuous transition, to a point where the periodic structure of slip-stick is difficult to identify and the flow becomes continuous as indicated in figure 5 for $H_{c}=0.7 \mathrm{~m}$.

The amplitude of the sound emission, for both sand and ballotini particles, was higher in the glass tube compared with the perspex tube. An interesting observation was made with regards to the sound duration in the glass tube: the honking time for sand shortened, while it lasted longer for ballotini particles.

\subsection{Travelling waves}

Regions of slip-stick motion propagate upwards through the tube, in a motion resembling a wave as illustrated in figure 3. For a given height, there is a gradual velocity increase followed by a rapid deceleration. This can be explained by a gradual expansion of packing fraction, where particles fall into gaps left by the movement of lower particles, followed by a sudden stop. In figure 6 we show we 


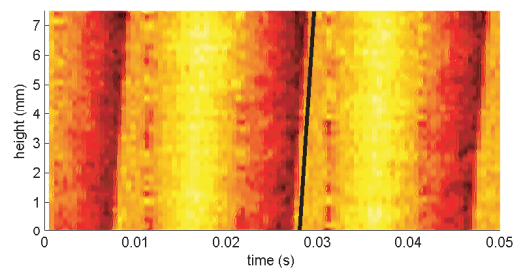

Figure 6. Waves of motion (red) propagate upwards through the imaged region extremely rapidly $\left(\approx 60 \mathrm{~ms}^{-1}\right)$, as seen from the steep gradient of these lines.

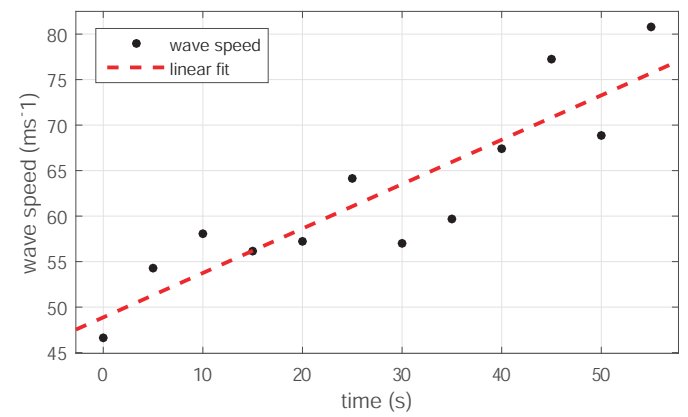

Figure 7. Wave speed $c$ increases linearly with time t, $c=A t+b$, with $A=0.49 m s^{-2}, b=48.9 \mathrm{~ms}^{-1}$, coefficient of determination $R^{2}=0.80$.

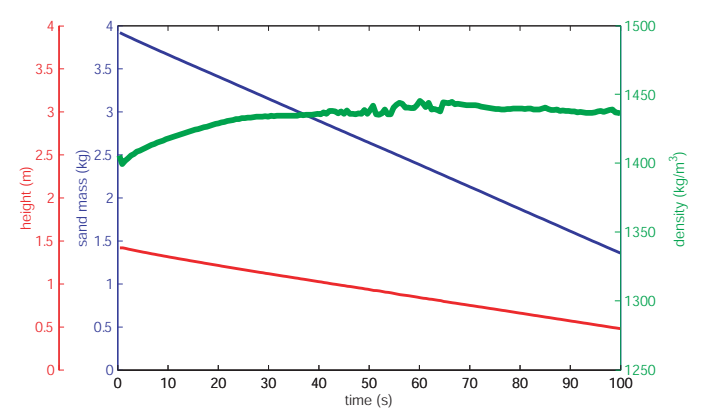

Figure 8. Mean surface height, particle mass, and density during the flow of sand particles in a perspex tube through a gap width of $10 \mathrm{~mm}$.

can estimate the speed of this slip-stick wave $c$, which is distinct from the particle speed $u_{a}$ and $u_{m}$, by looking at the phase shift between particle velocity peaks at each end of the imaged region Using two cameras at a separation of $50 \mathrm{~cm}$, we obtain a more accurate measure of mean wave speed over the whole tube. Locally, wave speed is known to vary with height [4], but average speed provides a more important measurement for setting the frequency. For 0.3$0.4 \mathrm{~mm}$ ballotini, the wave speed increased during the flow by a factor of 2, as shown in figure 7 . During this time, the honking frequency also increases, but not as rapidly. A central question is therefore what determines the length scale of the slip-stick.

One unknown factor is the packing fraction, which changed significantly during the flow. The wave speed in granular materials scales with the pressure as $p^{1 / 6}$, with pressure depending on both density and overburden height. The flow rate at the outflow point is constant, as confirmed by measurements from the scale. The height of the bulk surface, however, does not decrease at a constant rate. Initially, the height drops more rapidly during the first third of the flow, after which the level drops at a constant rate as illustrated in figure 8 . This corroborates evidence from particle tracking where the average particle velocity was higher at the top of the tube, implying a lower average density here. The changing height but constant outflow rate results in an increasing packing fraction and density during the first third of the flow duration, after which the packing fraction stabilizes, providing another factor for increasing wave speed and increasing resonant frequency over time.

\section{Conclusion}

We find that the slip-stick motion and the emitted acoustical frequency are strongly correlated with each other: the repetitive frequency of slip-stick is equal to the frequency of the sound produced by the structural resonance in the tube during discharge. The upward travelling waves, connected to a convective linear stability in [5], create regions where active slip-stick occurs. The key open question is what the effect is of the frictional characteristics between the different grains and the tube choices, and how this quantitatively sets the frequency of slip-stick motion - further work is necessary to answer this question.

We would like to acknowledge support by Dr. Marc Masen (Imperial College London) and Professor André de Boer (University of Twente) at earlier stages of this project, and the collaboration with Professor Jim Woodhouse (University of Cambridge) on the deployment of accelerometers. NMV would like to acknowledge financial support from the Royal Society Dorothy Hodgkin Research Fellowship RG70273 and KW support from a LMS summer bursary and the Bridgewater programme.

\section{References}

[1] K. Wilde, J. Tejchman, M. Rucka, and M. Niedostatkiewicz, Powder Technol. 198, 38 (2010)

[2] E. Porte, M. Masen, N.M. Vriend and A. de Boer, APS-DFD conference abstract, BAPS.2015.DFD.L10.12, (2015)

[3] J. M. Buick, J. Chavez-Sagarnaga, Z. Zhong, J. Y. Ooi, Pankaj, D. M. Cambell, and C. A. Greated, J. Engrg. Mech. 131, 299 (2005)

[4] T. Börzsönyi and Z. Kovacs, Phys. Rev. E 83, 03230 (2011)

[5] L. Bonneau and T. Catelin-Jullien, B. Andreotti, Phys. Rev. E 82, 011309 (2010)

[6] J.W.S. Rayleigh, The Theory of Sound, (Dover, London, 1945)

[7] K. Wilde, M. Rucka, and J. Tejchman, Powder Technol. 186, 113 (2008)

[8] J. M. Buick, Pankaj, J. Y. Ooi, J. Chavez-Sagarnaga, A. Pearce, G. Houghton, J. Phys D: Appl. Phys. 37, 2755-2760 (2004) 